\title{
A MIXED FRAMEWORK FOR TOPOLOGICAL MODEL REDUCTION OF COUPLED PDES
}

\author{
Ingeborg G. Gjerde*, Marie E. Rognes*,1 \\ *Simula Research Laboratory \\ 1364, Fornebu, Norway \\ e-mail: ingeborg@simula.no, meg@simula.no \\ ${ }^{1}$ University of Bergen \\ 5007, Bergen, Norway
}

Key words: multiscale, mixed finite-elements

\begin{abstract}
In this work, we consider a set of mixed-dimensional PDEs that are used to model e.g. microcirculation, root water uptake and the flow of fluids in a reservoir perforated with wells. To be more precise, we consider here the Poisson equation posed in two distinct domains. The two are then coupled by the use of a filtration law. We show how the mixed framework is a natural setting for this problem, as it allows the two equations to be posed using global variables. Further, the applications we consider are characterized by a scale disparity between the two domains. With this in mind, we perform a physically motivated averaging of the coupling condition. This has the advantage of allowing the solution to be approximated using non-conforming, coarse meshes.
\end{abstract}

\section{Introduction}

We consider a system of coupled elliptic partial differential equations (PDEs) describing flow in a porous medium perforated by thin channels. These equations can model a range of interesting and important phenomena including blood flow in vascularized tissue $[10,18$, $3]$ or fluid flow in wells drilled through the subsurface $[19,1,4]$. Here, we are interested in applications characterized by a scale disparity between the medium and the channels: the channels have a negligible radius compared to the size of the full domain. Discretizing the full system accurately typically relies on resolving the channels geometrically, a meshing 
restriction that may lead to prohibitively expensive simulations. An alternative and in many cases preferable approach is to introduce topological model reduction and reduce the channel representation to the one-dimensional centerlines. The resulting system of equations is often referred to as a coupled $1 D-3 D$ model.

Coupled 1D-3D flow models were first derived by D'Angelo [6, Section 3.2] and analyzed by D'Angelo and Quarteroni in the early 2000s [5]. Their derivation gives rise to a line source $\delta_{\Lambda}$ coupling the $1 \mathrm{D}$ model to the $3 \mathrm{D}$ model. As the dimensional gap between the two models is larger than one, this has the unfortunate consequence of inducing a singularity in the solution. Subsequent analyses of these models and solutions have relied on weighted Sobolev spaces [7]. Finite element approximations of such solutions will fail to converge in the $H^{1}(\Omega)$-norm, but converge in a weaker weighted $H_{\alpha}^{1}(\Omega)$-norm. The use of norms weighted with respect to the distance to the line can be interpreted as giving up control over the solution around the line. Several strategies have been proposed to deal with the associated numerical challenges. Kuchta et al [14] developed preconditioners suitable for the coupled 1D-3D problem; Koch et al [11] used a smoothing kernel to distribute the line source over a 3D subdomain; and in [9], a splitting scheme was formulated such that a smooth remainder term is approximated rather than the full solution.
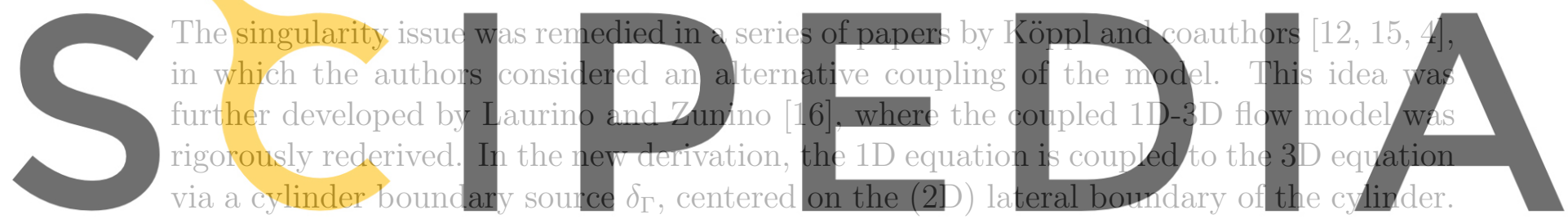

The result is a 1D-(2D)-3D method where the dimensional gap has been reduced to 1 .

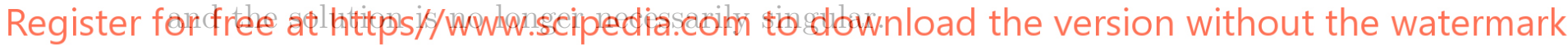

In this work, we consider a mixed formulation of the coupled 1D-3D flow model including both the pressures and fluxes as unknowns. Comparatively little work has been done on this formulation compared to the primal one in the context of topological model reduction. As the first exception, we note [17], where the authors take the strong formulation of the coupled 1D-3D model (with a line source [5]) as their starting point. They then directly reformulate it as a set of mixed equations in 1D and 3D. More recently, an extended finite element method was formulated for the mixed coupled 1D-3D flow model by Březina and Exner [2]. They take, as their starting point, the strong formulation of the coupled 1D3D flow model (with a cylinder source [16]), before directly reformulating it as a mixed equation in $1 \mathrm{D}$ and $3 \mathrm{D}$.

In this article, we derive a mixed formulation of the coupled 1D-3D flow model, taking the same set of model equations as in [16] as our starting point. Moreover, we follow their procedure of first deriving the variational formulation of the problem and then performing the averaging that leads to a dimensional reduction. We show that the mixed framework 
gives a distinctive setting for the problem, as it allows a natural formulation of the model via global variables defined over the two flow domains [13]. Moreover, this allows for a straightforward averaging of the coupling condition. Interestingly, the model we derive here differs characteristically from the mixed coupled 1D-3D model studied in [17]. It shares several similarities with the mixed model postulated in [2], particularly in the coupling. Their model, however, introduces an additional equation for the 1D domain. In our model, this is not a prerequisite as the formulation uses a global variable.

\section{Mathematical model formulation}

\subsection{Geometrical setting and scale disparity assumption}

Consider a given open domain $\Omega \subset \mathbb{R}^{3}$, bounded and convex with smooth boundary $\partial \Omega$. Embedded in this domain we have a generalized cylinder $\Omega_{i}$, defined as the swept volume of a circle of radius $R>0$ along a curve $\Lambda$. This gives rise to the perforated domain $\Omega_{e}=\Omega \backslash \Omega_{i}$. The cylinder $\Omega_{i}$ is assumed to have a $C^{2}$-regular centreline $\Lambda$, parametrized by $\lambda(s)=\left[\lambda^{1}(s), \lambda^{2}(s), \lambda^{3}(s)\right]$ such that $\Lambda=\{\lambda(s)\}$ for $s \in(0, L)$. Here, we assume
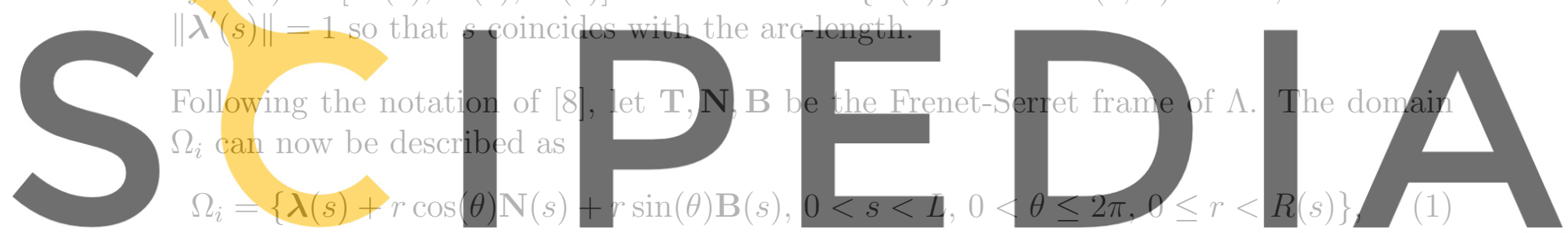

where $r=r(s)$ and $\theta=\theta(s)$ are the cylindrical coordinates of the local coordinate system

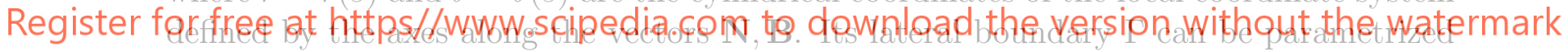
by

$$
\Gamma=\{\boldsymbol{\lambda}(s)+R(s) \cos (\theta) \mathbf{N}(s)+R(s) \sin (\theta) \mathbf{B}(s), 0<s<L, 0<\theta \leq 2 \pi\},
$$

and its top and bottom boundaries, denoted $\Gamma_{0} \cup \Gamma_{L}$, by

$$
\begin{aligned}
& \Gamma_{0}=\{\boldsymbol{\lambda}(0)+r(0) \cos (\theta) \mathbf{N}(0)+r(0) \sin (\theta) \mathbf{B}(0), 0<\theta \leq 2 \pi, 0 \leq r<R(0)\}, \\
& \Gamma_{L}=\{\boldsymbol{\lambda}(L)+r(L) \cos (\theta) \mathbf{N}(L)+r(L) \sin (\theta) \mathbf{B}(L), 0<\theta \leq 2 \pi, 0 \leq r<R(L)\} .
\end{aligned}
$$

For convenience, we assume that $\Gamma_{0} \cup \Gamma_{L} \subset \partial \Omega$, i.e. that the top and bottom boundaries of the cylinder coincide with the boundary of $\Omega$.

Let $\mathcal{D}(s)=\left[x(r, t), y(r, t):(0, R(s)) \times(0, T(s)) \rightarrow \mathbb{R}^{2}\right]$ be a parametrization of the cross section of $\Omega_{i}$. Taking $|\cdot|$ to denote the Lebesgue measure of a set, $|\partial \mathcal{D}(s)|$ then denotes the circumference of the cross-section of $\Omega_{i}$ at $\boldsymbol{\lambda}(s)$.

Finally and throughout, we make the following fundamental assumption of scale disparity between $\Omega$ and $\Omega_{i}$ : 

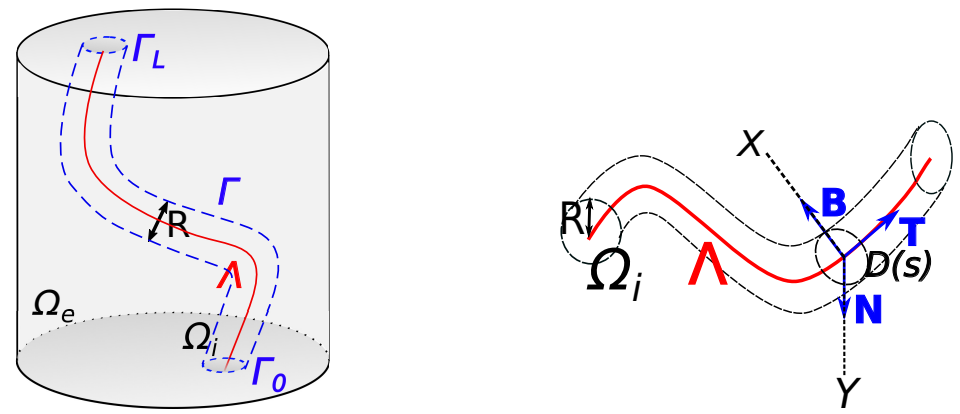

Figure 1: (Left) The domain $\Omega$ embedded with a generalized cylinder $\Omega_{i}$. The cylinder $\Omega_{i}$ is described by a centreline $\Lambda$ and has a radius $R$. (Right) A generalized cylinder $\Omega_{i}$ with centreline $\Lambda$ and radius $R$. The curve $\Lambda$ is associated with a Frenet-Serret frame $\mathbf{T}, \mathbf{N}, \mathbf{B}$; here, $\mathbf{T}$ denotes its unit tangent vector, $\mathbf{n}$ its unit normal vector, and $\mathbf{B}$ its unit binormal vector.

\section{A0: The transversal diameter $R(s)$ of $\Omega_{i}$ is small compared to the diameter of $\Omega$.}

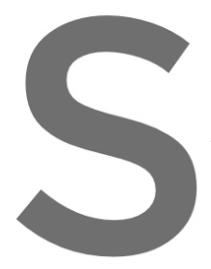

\subsection{Governing equation \\ We here consider the sisting of Darcy flow equations in $\Omega_{i}$ and $\Omega_{e}$
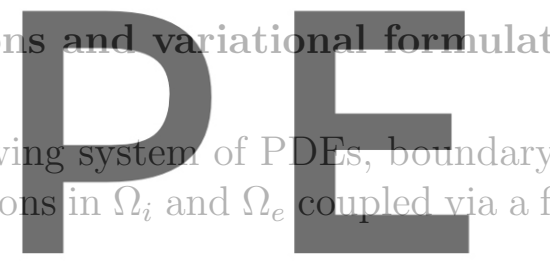
for $*=i$.e such that
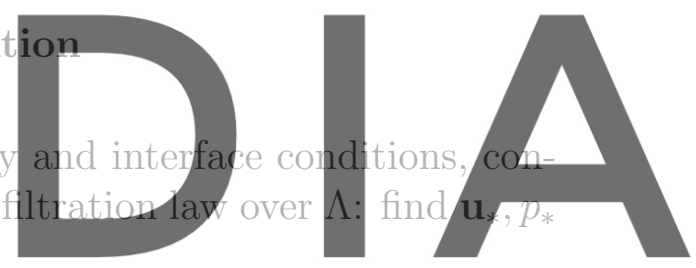

Register for free at https/AwWw.sËipediz.com to download theiversion without the watermark

$$
\begin{aligned}
\nabla \cdot \mathrm{u}_{*} & =f_{*} & & \text { in } \Omega_{*}, \\
\llbracket \mathbf{u} \cdot \mathbf{n} \rrbracket & =0 & & \text { on } \Gamma, \\
\mathbf{u}_{i} \cdot \mathbf{n}_{i} & =\beta \llbracket p \rrbracket & & \text { on } \Gamma, \\
\mathbf{u}_{i} \cdot \mathbf{n}_{i} & =g & & \text { on } \Gamma_{0} \cup \Gamma_{L}, \\
\mathbf{u}_{e} \cdot \mathbf{n}_{e} & =g & & \text { on } \partial \Omega_{e},
\end{aligned}
$$

where $\llbracket p \rrbracket=p_{i}-p_{e}$ denotes the pressure jump across $\Gamma, \llbracket \mathbf{u} \cdot \mathbf{n} \rrbracket=\mathbf{u}_{i} \cdot \mathbf{n}_{i}-\mathbf{u}_{e} \cdot \mathbf{n}_{e}$ is the jump in normal flux across $\Gamma, \kappa_{*} \in L^{\infty}\left(\Omega_{*}\right)$ is a uniformly positive permeability of the domain $\Omega_{*}, g \in H^{\frac{1}{2}}(\partial \Omega)$ is a given boundary function on $\partial \Omega$ and $\beta \in L^{\infty}(\Gamma)$ is a uniformly positive permeability of the interface $\Gamma$. Following up on assumption (A0), we assume the interface permeability to be radially symmetric such that $\beta=\beta(s)$.

In this work, we turn to study a dual mixed formulation of this model, which can be derived as follows. Let $\mathcal{I}_{i}$ and $\mathcal{I}_{e}$ denote the indicator functions for $\Omega_{i}$ and $\Omega_{e}$, respectively. Let $\mathbf{u}$ and $p$ denote the global variables $\mathbf{u}=\mathcal{I}_{i} \mathbf{u}_{i}+\mathcal{I}_{e} \mathbf{u}_{e}$ and $p=\mathcal{I}_{i} p_{i}+\mathcal{I}_{e} p_{e}$. Similarly, we 
take $\kappa=\mathcal{I}_{i} \kappa_{i}+\mathcal{I}_{e} \kappa_{e}$ and $f=\mathcal{I}_{i} f_{i}+\mathcal{I}_{e} f_{e}$. Let

$$
\begin{aligned}
V_{g} & =\left\{\mathbf{v} \in H(\operatorname{div} ; \Omega): \mathbf{v} \cdot \mathbf{n} \in L^{2}(\Gamma), \mathbf{v} \cdot \mathbf{n}=g \text { on } \partial \Omega\right\}, \\
Q & =L^{2}(\Omega),
\end{aligned}
$$

and take test functions $\mathbf{v} \in V_{0}$ and $q \in Q$. Multiplying (5a)-(5b) by $\mathbf{v}$ and $q$, respectively, and integrating by parts, we obtain

$$
\begin{aligned}
\sum_{* \in\{i, e\}}\left(\kappa_{*}^{-1} \mathbf{u}_{*}, \mathbf{v}_{*}\right)_{\Omega_{*}}-\left(\nabla \cdot \mathbf{v}_{*}, p_{*}\right)_{\Omega_{*}}+\left(p_{*}, \mathbf{v}_{*} \cdot \mathbf{n}_{*}\right)_{\partial \Omega_{*}} & =0 \\
\sum_{*=\{i, e\}}\left(\nabla \cdot \mathbf{u}_{*}, q_{*}\right) \Omega_{*} & =\sum_{*=\{i, e\}}\left(f_{*}, q_{*}\right)_{\Omega_{i}} .
\end{aligned}
$$

The integration by parts picks up boundary terms defined on $\bigcup_{* \in\{i, e\}} \partial \Omega_{*}=\Gamma \cup \Gamma_{0} \cup \Gamma_{L} \cup$ $\partial \Omega_{e}$. On $\Gamma_{0}$ and $\Gamma_{L}$, we have $\mathbf{v}_{i} \cdot \mathbf{n}_{i}=0$ by $\mathbf{v} \in V_{0}$. Similarly, on $\partial \Omega_{e}, \mathbf{v}_{e} \cdot \mathbf{n}_{e}=0$. Thus, these boundary terms vanish everywhere except for on the lateral cylinder boundary $\Gamma$. As $\mathbf{v} \in X \subset H(\operatorname{div} ; \Omega)$, we have $\mathbf{v}_{i} \cdot \mathbf{n}_{i}=-\mathbf{v}_{e} \cdot \mathbf{n}_{e}$. Thus,

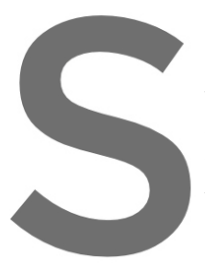

$$
\sum\left(p_{*}, \mathbf{v}_{*} \cdot \mathbf{n}_{i}\right)_{\Gamma}=\left(\left(p_{e}-p_{i}\right), \mathbf{v}_{e} \cdot \mathbf{n}_{e}\right)_{\Gamma}=\left(\beta^{-1} \mathbf{u}_{e} \cdot \mathbf{n}_{e}, \mathbf{v}_{e} \cdot \mathbf{n}_{e}\right)_{\Gamma} .
$$

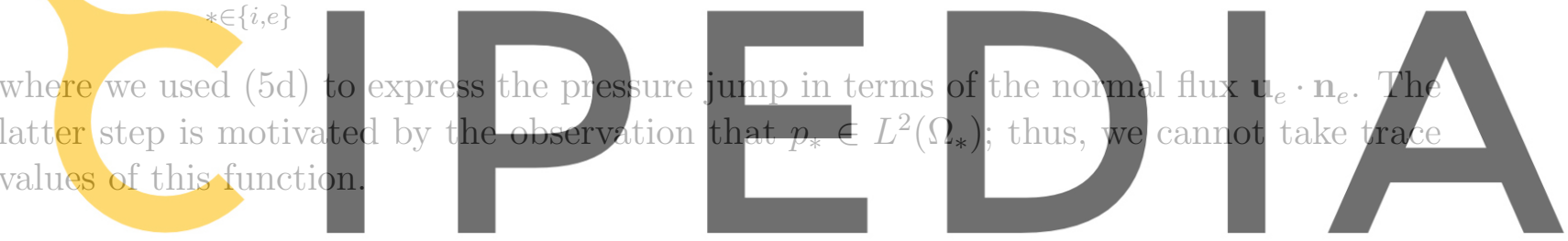

The dual mixed formulation can then be written in terms of the global variables as follows:

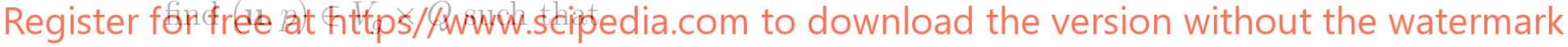

$$
\begin{aligned}
\left(\kappa^{-1} \mathrm{u}, \mathrm{v}\right)_{\Omega}+\left(\beta^{-1} \mathrm{u} \cdot \mathrm{n}, \mathrm{v} \cdot \mathrm{n}\right)_{\Gamma}-(\nabla \cdot \mathrm{v}, p)_{\Omega} & =0, \\
(\nabla \cdot \mathbf{u}, q)_{\Omega} & =(f, q)_{\Omega},
\end{aligned}
$$

for all $(\mathbf{v}, q) \in V_{0} \times Q$. The choice of $\mathbf{n}=\mathbf{n}_{i}$ or $\mathbf{n}=\mathbf{n}_{e}$ is arbitrary; we set $\mathbf{n}=\mathbf{n}_{i}$. We remark that the formulation is not well posed when defined over $H(\operatorname{div} ; \Omega) \times L^{2}(\Omega)$. In this latter case, one has that $\mathbf{u} \cdot \mathbf{n}, \mathbf{v} \cdot \mathbf{n} \in H^{-\frac{1}{2}}(\Gamma)$, meaning that the term $(\mathbf{u} \cdot \mathbf{n}, \mathbf{v} \cdot \mathbf{n})_{\Gamma}$ may not be bounded. This is the reason we restrict $\mathbf{u}, \mathbf{v} \in V$ wherein $\mathbf{v} \cdot \mathbf{n}, \mathbf{u} \cdot \mathbf{n} \in L^{2}(\Gamma)$. Such a formulation is well-posed, as demonstrated by Theorem 1 below.

Theorem 1. The variational formulation (8) is well-posed over its function spaces (7).

Proof. For the sake of simplicity, we assume $g=0$ and set $V=V_{0}$. Define

$$
\begin{aligned}
a(\mathbf{u}, \mathbf{v}) & =\left(\kappa^{-1} \mathbf{u}, \mathbf{v}\right)_{\Omega}+(\mathbf{u} \cdot \mathbf{n}, \mathbf{v} \cdot \mathbf{n})_{\Gamma}, \\
b(\mathbf{v}, p) & =-(p, \nabla \cdot \mathbf{v})_{\Omega},
\end{aligned}
$$


and $L(q)=(f, q)_{\Omega}$. Then (8) can be written as: find $(\mathbf{u}, p) \in V \times Q$ such that

$$
\begin{aligned}
a(\mathbf{u}, \mathbf{v})+b(\mathbf{v}, p) & =0 \\
b(\mathbf{u}, q) & =L(q)
\end{aligned}
$$

for all $(\mathbf{v}, q) \in V \times Q$. The proof now is by verifying the Ladyzhenskaya-Babuška-Brezzi conditions. Firstly, $a$ and $b$ are both bounded on the space $V \times Q$ by definition. Moreover, $a$ is symmetric and coercive on the kernel $K$ of $Q$, defined as

$$
K=\{\mathbf{v}: b(\mathbf{v}, p)=0 \quad \forall p \in Q\} .
$$

To see this, note that for any fixed $\mathbf{v} \in K$, one can set $p=\nabla \cdot \mathbf{v} \in Q$, which yields

$$
b(\mathbf{v}, \nabla \cdot \mathbf{v})=\|\nabla \cdot \mathbf{v}\|_{L^{2}(\Omega)}^{2}=0 .
$$

\section{It follows that}

$$
a(\mathbf{v}, \mathbf{v})=(\mathbf{v}, \mathbf{v})_{\Omega}+(\mathbf{v} \cdot \mathbf{n}, \mathbf{v} \cdot \mathbf{n})_{\Gamma}=\|\mathbf{v}\|_{L^{2}(\Omega)}^{2}+\|\nabla \cdot \mathbf{v}\|_{L^{2}(\Omega)}^{2}+\|\mathbf{v} \cdot \mathbf{n}\|_{L^{2}(\Gamma)}^{2} \equiv\|\mathbf{v}\|_{V}^{2},
$$

\section{meaning that $a$ is indeed coercive on the kernel of $b$}
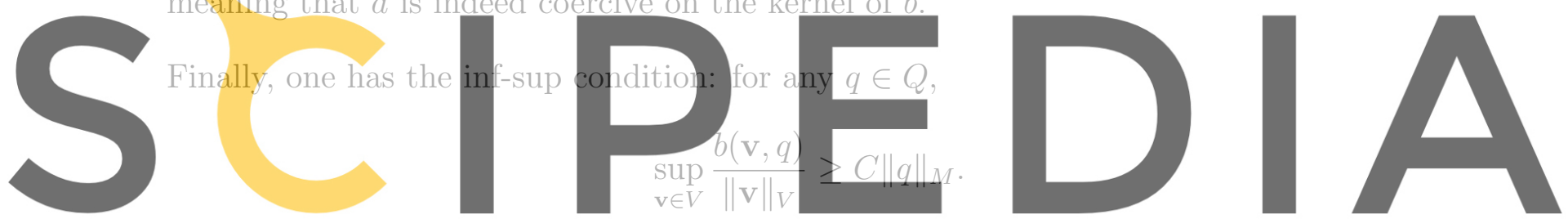

To see this, take $\mathbf{v}$ such that. $\nabla \cdot \mathbf{v}_{\mathbf{v}}=q$. For $\mathbf{v}=-\nabla \xi$, this reduces to solving $-\Delta \xi=q$. As

Register for freesat https/Lwww sccipedia.con to downhoad the version without the watermark

has $T_{\Gamma} v_{i} \in H^{\frac{1}{2}}(\Gamma)$, where $T_{\Gamma}$ denotes the trace operator on $\Gamma$ and $v_{i}$ denotes a component

of $\mathbf{v}$. Assuming that $\Omega_{i}$ is smooth enough for $\mathbf{n}_{i}$ to be sufficiently smooth, one then has $\mathbf{v} \cdot \mathbf{n}=\sum_{i} v_{i} n_{i} \in L^{2}(\Gamma)$, and so one indeed has $\mathbf{v} \in V$. Moreover,

$$
\|\xi\|_{H^{2}(\Omega)}=\|\mathbf{v}\|_{\left(H^{1}(\Omega)\right)^{3}} \leq C_{S}\|q\|_{Q}
$$

where $C_{S}$ denotes a stability constant, meaning that

$$
\begin{aligned}
\|\mathbf{v}\|_{V}^{2} & =\|\mathbf{v}\|_{H(\operatorname{div} ; \Omega)}^{2}+\|\mathbf{v} \cdot \mathbf{n}\|_{L^{2}(\Gamma)}^{2} \leq\|\mathbf{v}\|_{H(\operatorname{div} ; \Omega)}^{2}+C_{T}\|\mathbf{v}\|_{H(\operatorname{div} ; \Omega)}^{2} \\
& \leq\left(1+C_{T}\right)\|\mathbf{v}\|_{\left(H^{1}(\Omega)\right)^{3}}^{2}=C_{S}\left(1+C_{T}\right)\|q\|_{L^{2}(\Omega)},
\end{aligned}
$$

where $C_{T}$ denotes a trace constant. Inserting this $\mathbf{v}$, one finds

$$
\sup _{\mathbf{v} \in V} \frac{b(\mathbf{v}, q)}{\|\mathbf{v}\|_{V}} \geq \frac{(q, q)_{\Omega}}{\|\mathbf{v}\|_{V}} \geq \frac{\|q\|_{Q}^{2}}{C_{S}\left(1+C_{T}\right)\|q\|_{Q}} \geq \frac{1}{C_{S}\left(1+C_{T}\right)}\|q\|_{Q}
$$




\section{Dimensional reduction}

Recall now our fundamental assumption of a scale disparity: $R \ll \operatorname{size}(\Omega)$. Let $\Gamma_{h}$ and $\Omega_{h}$ denote discretizations of $\Gamma$ and $\Omega$, respectively. Upon approximation with finite elements, the formulation (8) may require conforming discretizations to converge, in the sense that $\Gamma_{h} \subset \mathcal{E}_{h}$ with $\mathcal{E}_{h}$ denoting the edges in $\Omega_{h}$. When $R \ll \operatorname{size}(\Omega)$, this requires a very fine discretization around $\Gamma$. This introduces a large number of degrees of freedom, ultimately making the full discretization prohibitively expensive to compute. In the reduced model, this is circumvented by introducing a physically motivated averaging of the coupling condition. The averaging reduces the coupling condition from $2 \mathrm{D}$ to $1 \mathrm{D}$; hence the name coupled 1D-3D flow model or reduced model. The averaging weakens the coupling condition, so that the discretizations $\Gamma_{h}$ and $\Omega_{h}$ no longer have to conform and thus the reduced flow equations can be discretized using coarser meshes.

\section{Let $\Pi_{\partial \mathcal{D}}$ denote the averaging operator over $\partial \mathcal{D}$, with the averaged variable $\bar{q}$ defined as}

$$
\bar{q}(s)=\Pi_{\partial \mathcal{D}}(q)=\frac{1}{|\partial \mathcal{D}|} \int_{0}^{2 \pi} R(s) q(s, \theta, R(s)) \mathrm{d} \theta .
$$

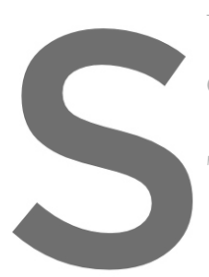

We note that the centreline $\Lambda$.

To present a reduced

A1: We have $\left\|p \rrbracket \approx \Pi_{\mathcal{D}}\right\| p \rrbracket$
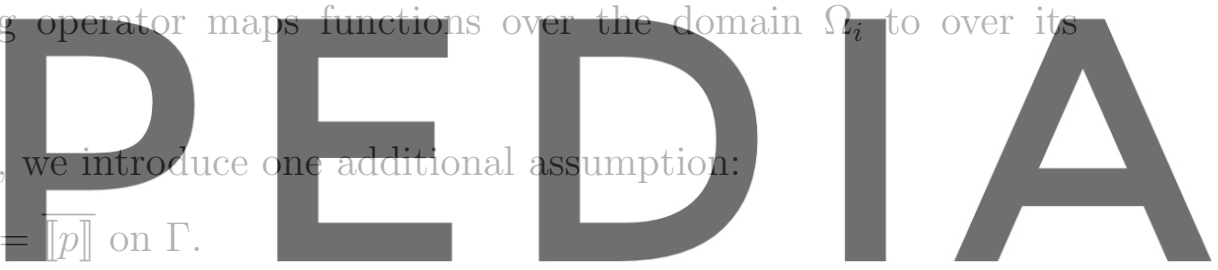

This can be interpreted as saying that, on each cross-section of $\Omega_{i}$, the pressure jump

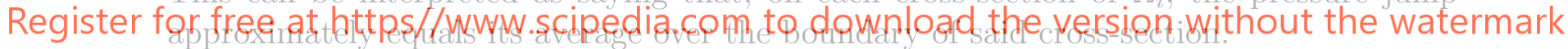

Under assumption A1, then

$$
(\beta \llbracket p \rrbracket, \mathbf{v} \cdot \mathbf{n})_{\Gamma} \approx \int_{\Lambda} \beta \overline{\llbracket p \rrbracket} \int_{\partial \mathcal{D}(s)} \mathbf{v} \cdot \mathbf{n} \mathrm{d} \theta \mathrm{d} s=|\partial \mathcal{D}|(\beta \overline{\llbracket p \rrbracket}, \overline{\mathbf{v} \cdot \mathbf{n}})_{\Lambda}
$$

Averaging both sides of the interface condition (5c) we find that $\overline{\mathbf{u}_{e} \cdot \mathbf{n}_{e}}=\beta \overline{\llbracket p \rrbracket}$. Thus

$$
(\beta \llbracket p \rrbracket, \mathbf{v} \cdot \mathbf{n})_{|\partial \mathcal{D}|, \Lambda} \approx\left(\beta^{-1} \overline{\mathbf{u} \cdot \mathbf{n}}, \overline{\mathbf{v} \cdot \mathbf{n}}\right)_{|\partial \mathcal{D}|, \Lambda},
$$

where we used the weighted norm notation $\left(|\partial \mathcal{D}| \beta^{-1} \overline{\mathbf{u} \cdot \mathbf{n}}, \overline{\mathbf{v} \cdot \mathbf{n}}\right)_{\Lambda}=\left(\beta^{-1} \overline{\mathbf{u} \cdot \mathbf{n}}, \overline{\mathbf{v} \cdot \mathbf{n}}\right)_{|\partial \mathcal{D}|, \Lambda}$.

These observations yield the following reduced model problem: find $(\mathbf{u}, p) \in \bar{V}_{g} \times Q$ such that

$$
\begin{aligned}
(\mathbf{u}, \mathbf{v})_{\Omega}+\left(\beta^{-1} \overline{\mathbf{u} \cdot \mathbf{n}}, \overline{\mathbf{v} \cdot \mathbf{n}}\right)_{|\partial \mathcal{D}|, \Lambda}- & (\nabla \cdot \mathbf{v}, p)_{\Omega}=0 \\
(\nabla \cdot \mathbf{u}, q)_{\Omega} & =(f, q)_{\Omega},
\end{aligned}
$$


for all $(\mathbf{v}, q) \in \bar{V}_{0} \times Q$, where

$$
\bar{V}_{g}=\left\{\mathbf{v} \in H(\operatorname{div} ; \Omega): \overline{\mathbf{v} \cdot \mathbf{n}} \in L^{2}(\Lambda), \mathbf{v} \cdot \mathbf{n}=g \text { on } \partial \Omega\right\},
$$

and $Q=L^{2}(\Omega)$ as before. A well-posedness result for the reduced model follows:

Theorem 2. The variational formulation (9) is well-posed.

Proof. The proof follows by the same steps as Theorem 1.

\section{Discretization}

For clarity, assume that the domain $\Omega$ is polyhedral, and admitting a decomposition $\mathcal{T}^{h}$ into tetrahedra $K$. As before, we have $\Omega_{i}$ denoting the generalized cylinder with centreline $\Lambda$ and radius $R$, and the perforated domain $\Omega_{e}$. For $\Omega_{i}$ and $\Omega_{e}$, we introduce the decompositions $\mathcal{T}_{i}^{h}$ and $\mathcal{T}_{e}^{h}$, respectively. Thus, $\Omega_{e}^{h}=\Omega^{h} \backslash \Omega_{i}^{h}$. We let $h, h_{i}, h_{e}$ denote the mesh sizes

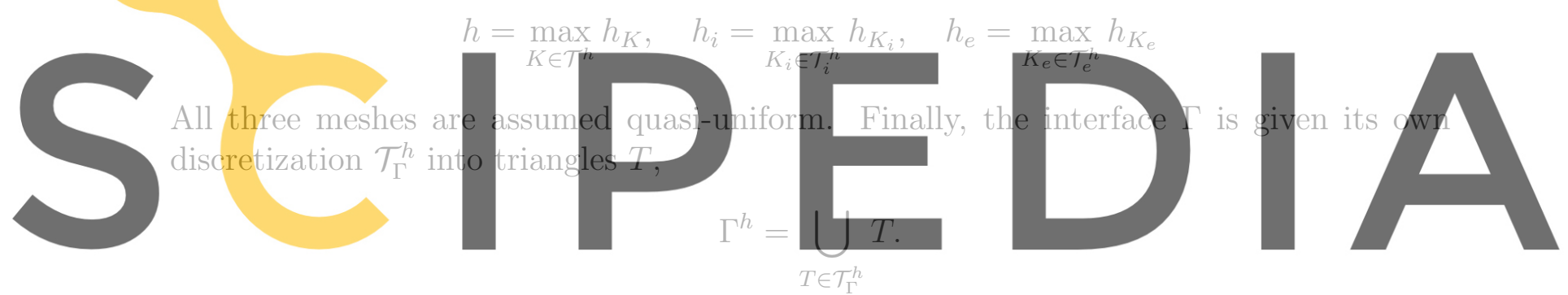

Register for free atehttps/hwyw scipedia, com to domnload the version without the watermark

up $\Gamma_{h}$ are facets $\mathcal{E}_{h}$ in $\Omega_{i}$ and $\Omega_{e}$.

For the discretization, we consider the discontinuous Lagrange elements of degree 0 to approximate $p$,

$$
D G_{h}^{0}\left(\Omega_{h}\right):=\left\{w_{h} \in L^{2}(\Omega):\left.w_{h}\right|_{K} \in P_{0}(K) \quad \forall K \in \mathcal{T}_{h}\right\},
$$

and the $H(\operatorname{div} ; \Omega)$-conforming Raviart-Thomas elements of degree 1 to approximate $\mathbf{u}$,

$$
R T_{h}^{1}\left(\Omega_{h}\right):=\left\{\mathbf{w}_{h} \in\left(L^{2}(\Omega)\right)^{3}:\left.\mathbf{w}_{h}\right|_{K} \in\left(P_{0}(K)\right)^{3} \oplus \mathbf{x} P_{0}(K) \quad \forall K \in \mathcal{T}_{h}\right\} .
$$

The mixed finite element discretization of the full model then reads: find $\mathbf{u}_{h} \in R T_{h}^{1}\left(\Omega_{h}\right)$ and $p_{h} \in D G_{h}^{0}\left(\Omega_{h}\right)$ such that

$$
\begin{aligned}
\left(\kappa^{-1} \mathbf{u}_{h}, \mathbf{v}_{h}\right)_{\Omega_{h}}+\left(\beta^{-1} \mathbf{u}_{h} \cdot \mathbf{n}_{h}, \mathbf{v}_{h} \cdot \mathbf{n}_{h}\right)_{\Gamma_{h}}-\left(\nabla \cdot \mathbf{v}_{h}, p_{h}\right)_{\Omega_{h}} & =0, \\
\left(\nabla \cdot \mathbf{u}_{h}, q_{h}\right)_{\Omega_{h}} & =\left(f, q_{h}\right)_{\Omega_{h}},
\end{aligned}
$$


for all $\mathbf{v}_{h} \in R T_{h}^{1}\left(\Omega_{h}\right)$ and $q_{h} \in D G_{h}^{0}\left(\Omega_{h}\right)$.

The mixed finite element discretization of the reduced model similarly reads: find $\mathbf{u}_{h} \in$ $R T_{h}^{1}\left(\Omega_{h}\right)$ and $p_{h} \in D G_{h}^{0}\left(\Omega_{h}\right)$ such that

$$
\begin{aligned}
\left(\kappa^{-1} \mathbf{u}_{h}, \mathbf{v}_{h}\right)_{\Omega_{h}}+\left(\beta^{-1} \overline{\mathbf{u}_{h} \cdot \mathbf{n}}, \overline{\mathbf{v}_{h} \cdot \mathbf{n}}\right)_{\Lambda_{h}}-(\nabla \cdot \mathbf{v}, p)_{\Omega_{h}} & =0, \\
\left(\nabla \cdot \mathbf{u}_{h}, q_{h}\right)_{\Omega_{h}} & =\left(f, q_{h}\right)_{\Omega_{h}},
\end{aligned}
$$

again for all $\mathbf{v}_{h} \in R T_{h}^{1}\left(\Omega_{h}\right)$ and $q_{h} \in D G_{h}^{0}\left(\Omega_{h}\right)$.

\section{$5 \quad$ Numerical experiments}

In this section, we will perform numerical experiments to investigate the approximation properties for the full (11) and reduced (12) mixed finite element discretizations. To this end, let $R=1, k_{*}=1$ for $* \in\{i, e\}, \beta=1$ and

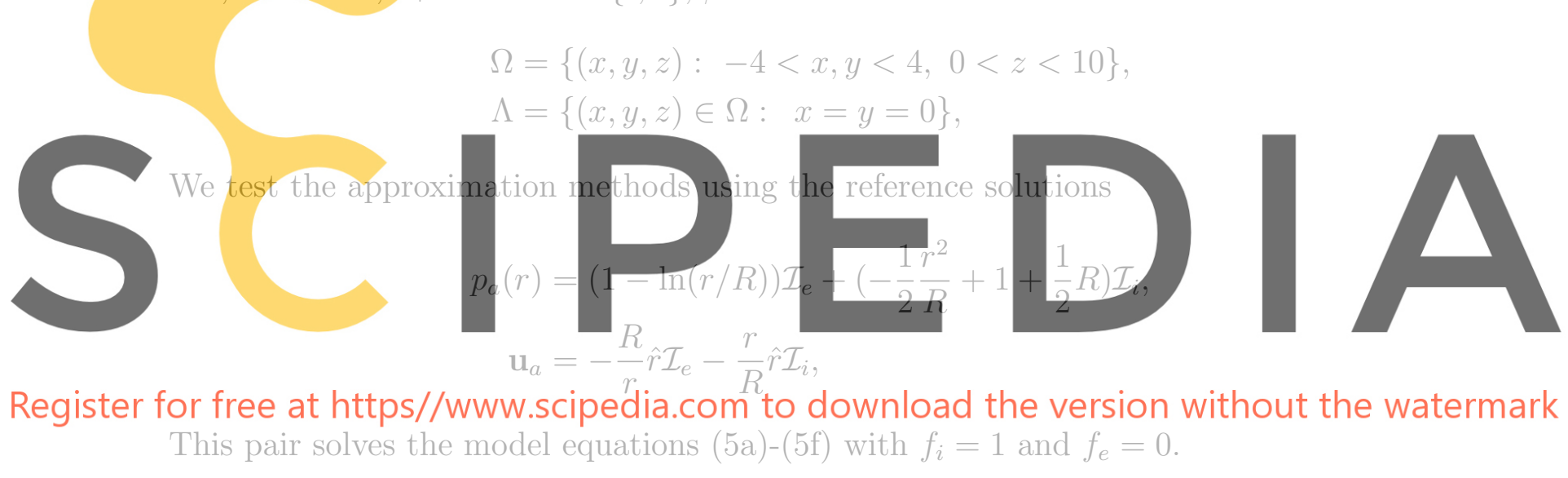

Figure 2 shows the full and reduced mixed finite element approximations of the reference solution. In Table 1, we give the convergence rates of each method. The full formulation is discretized on a conforming mesh. We have observed that this is necessary in order for the approximation to converge. Convergence rates for this method are listed in Table 1a. The convergence rate is calculated for each refinement. We also list the arithmetic average of these rates. Based on this, the pressure and flux approximations appear to converge optimally in the $L^{2}(\Omega)$-norm (i.e. with order 1 ). The flux approximation also appears to converge with order 1 in the $H(\operatorname{div} ; \Omega)$-norm.

The reduced formulation is seen to converge even though it uses a non-conforming mesh. Convergence rates for this method are listed in Table 1b. The pressure and flux approximations similarly appear to converge optimally in the $L^{2}(\Omega)$-norm. The flux approximation does not appear to converge optimally in the $H(\operatorname{div} ; \Omega)$-norm. 

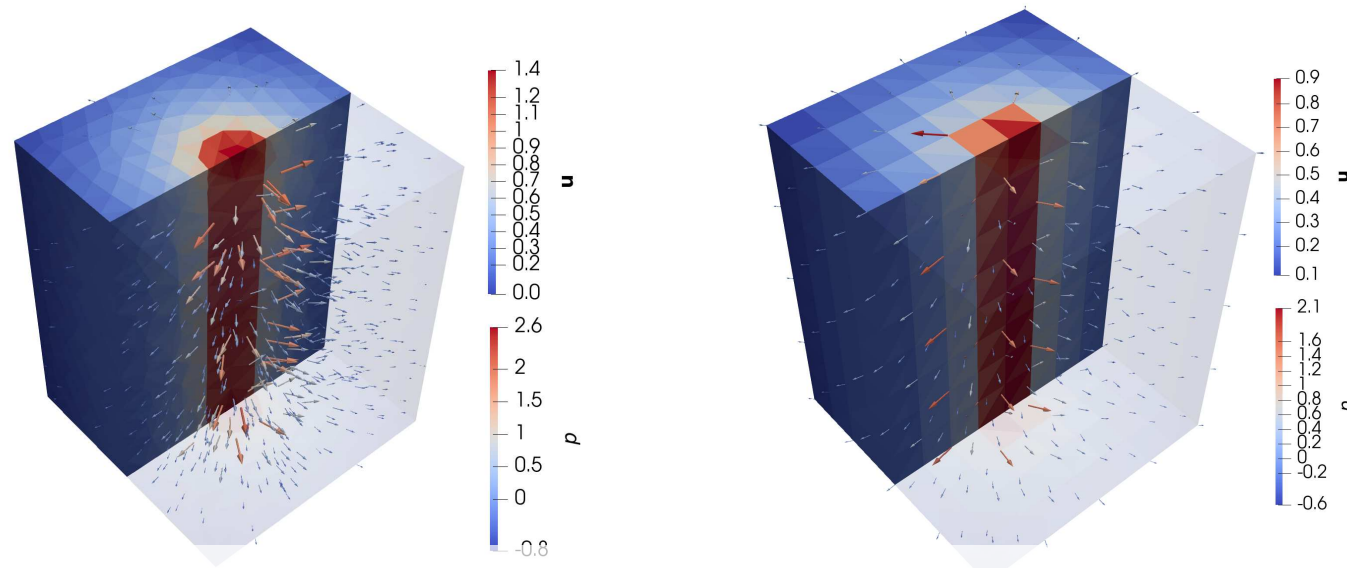

Figure 2: (Left) full model approximation on conforming mesh and (right) reduced approximation on non-conforming mesh.

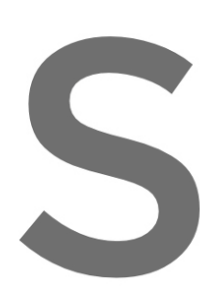

Table 1: Errors an

approximations, on

convergence rate is dalcher

refinement.
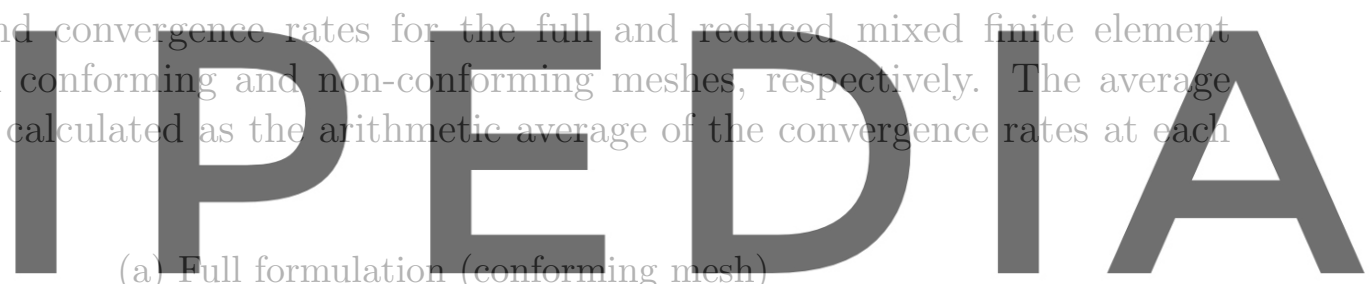

Register for free at https//www.scipedia.com to download the version without the watermark

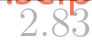

1.49

0.80

avg. rate: 1.2

(b) Averaged formulation (non-conforming mesh)

\begin{tabular}{llll}
$h$ & $\left\|p_{e}\right\|_{L^{2}(\Omega)}$ & $\left\|\mathbf{u}_{e}\right\|_{L^{2}(\Omega)}$ & $\left\|\mathbf{u}_{e}\right\|_{H(\mathrm{div} ; \Omega)}$ \\
\hline \hline 3.77 & 7.6445 & 6.2049 & 11.3195 \\
1.89 & $4.8369(0.7)$ & $2.7250(1.2)$ & $5.5505(1.0)$ \\
0.94 & $2.5792(0.9)$ & $1.3719(1.0)$ & $3.6188(0.6)$ \\
0.47 & $1.5906(0.7)$ & $0.8590(0.7)$ & $2.7838(0.4)$ \\
\hline avg. rate: & 0.8 & 1.0 & 0.7 \\
\hline
\end{tabular}




\section{References}

[1] I. Aavatsmark and R.A. Klausen. Well index in reservoir simulation for slanted and slightly curved wells in 3d grids. SPE Journal, 8(01):41-48, March 2003.

[2] Jan Březina and Pavel Exner. Extended finite element method in mixed-hybrid model of singular groundwater flow. Mathematics and Computers in Simulation, issn 0378-4754, December 2020.

[3] L. Cattaneo and P. Zunino. A computational model of drug delivery through microcirculation to compare different tumor treatments. International Journal for Numerical Methods in Biomedical Engineering, 30(11):1347-1371, November 2014.

[4] Daniele Cerroni, Federica Laurino, and Paolo Zunino. Mathematical analysis, finite element approximation and numerical solvers for the interaction of 3d reservoirs with 1d wells. GEM - International Journal on Geomathematics, 10(1), January 2019.

[5] C. D'Angelo and A. Quarteroni. On the coupling of 1d and 3d diffusion-reaction equations: Application to tissue perfusion problems. Mathematical Models and Methods in Applied Sciences, 18(08):1481-1504, August 2008.

[6] Carlo D'Angelo. Multiscale modelling of metabolism and transport phenomena in living tissues. $\mathrm{PhD}$ thesis, 2007.

[7] Carlo D'Angelo. Finite element approximation of elliptic problems with dirac measure terms in weighted spaces: Applications to one- and three-dimensional coupled problems. SIAM Journal on Numerical Analysis, 50(1):194-215, January 2012.

[8] I. Gansca, W.F. Bronsvoort, G. Coman, and L. Tambulea. Self-intersection avoidance and integral properties of generalized cylinders. Computer Aided Geometric Design, 19(9):695 - 707, December 2002.

[9] Ingeborg G. Gjerde, Kundan Kumar, Jan M. Nordbotten, and Barbara Wohlmuth. Splitting method for elliptic equations with line sources. ESAIM: Mathematical Modelling and Numerical Analysis, 53(5):1715-1739, September 2019.

[10] L. Grinberg, E. Cheever, T. Anor, J. R. Madsen, and G. E. Karniadakis. Modeling blood flow circulation in intracranial arterial networks: A comparative $3 \mathrm{~d} / 1 \mathrm{~d}$ simulation study. Annals of Biomedical Engineering, 39(1):297-309, January 2011.

[11] Timo Koch, Martin Schneider, Rainer Helmig, and Patrick Jenny. Modeling tissue perfusion in terms of 1d-3d embedded mixed-dimension coupled problems with distributed sources. arXiv e-prints, page arXiv:1905.03346, 2019. 
[12] Tobias Köppl, Ettore Vidotto, Barbara I. Wohlmuth, and Paulo Zunino. Mathematical modelling, analysis and numerical approximation of second order elliptic problems with inclusions. Numerical Mathematics and Advanced Applications ENUMATH 2015, 2017.

[13] Miroslav Kuchta, Kent-André Mardal, and Marie E. Rognes. Solving the EMI equations using finite element methods. In Modeling Excitable Tissue, pages 56-69. Springer International Publishing, October 2020.

[14] Miroslav Kuchta, Magne Nordaas, Joris C. G. Verschaeve, Mikael Mortensen, and Kent-Andre Mardal. Preconditioners for saddle point systems with trace constraints coupling 2d and 1d domains. SIAM Journal on Scientific Computing, 38(6):B962B987, 2016.

[15] Tobias Köppl, Ettore Vidotto, Barbara Wohlmuth, and Paolo Zunino. Mathematical modeling, analysis and numerical approximation of second-order elliptic problems with inclusions. Mathematical Models and Methods in Applied Sciences, 28(05):953978, March 2018.

[16] Federica Laurino and Paolo Zunino. Derivation and analysis of coupled PDEs on manifolds with high dimensionality gap arising from topological model reduction. ESAIM: Mathematical Modelling and Numerical Analysis, 53(6):2047-2080, November 2019 .

[17] Domenico Notaro, Laura Cattaneo, Luca Formaggia, Anna Scotti, and Paolo Zunino. A Mixed Finite Element Method for Modeling the Fluid Exchange Between Microcirculation and Tissue Interstitium, pages 3-25. Springer International Publishing, Cham, 2016.

[18] Ettore Vidotto, Timo Koch, Tobias Köppl, Rainer Helmig, and Barbara Wohlmuth. Hybrid models for simulating blood flow in microvascular networks. Multiscale Modeling \& Simulation, 17(3):1076-1102, January 2019.

[19] Christian Wolfsteiner, Louis J. Durlofsky, and Khalid Aziz. Calculation of well index for nonconventional wells on arbitrary grids. Computational Geosciences, 7(1):61-82, Mar 2003. 